kuppeln alle $\mathrm{zu}$ weniger als $1^{\%} \%$ in Orthostellung in $\alpha$-Naphtol ${ }^{1}$ ): Die Alkalinität spielt keine grosse Rolle. 2,6-Dibromanilin (als reines Diazoniumsulfat verwendet) kuppelt wie folgt:

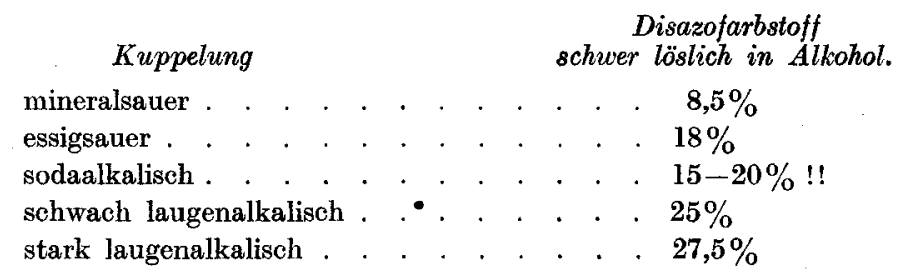

Zürich, Technisch-chemisches Laboratorium - der Eidg. Techn. Hochschule, März 1921.

\title{
Über die erschöpfende Sulfuration des Naphtalins
} von

\section{Hans Eduard Fierz und Fritz Sehmid.}

(31. III. 21.)

Armstrong und Wynne ${ }^{2}$ haben in einer Reihe von ausgezeichneten Untersuchungen gezeigt, dass Sulfogruppen in den Naphtalinkern niemals zueinander in ortho-, para- oder peri-Stellung eintreten können. Aus dieser Regel würde sich ergeben, dass bei der erschöpfenden Sulfuration des Naphtalins nur zwei Endprodukte auftreten können, nämlich die Trisulfosäure 1,3,6 (I) und die Tetrasulfosäure 1,3,5,7 (II):

I.

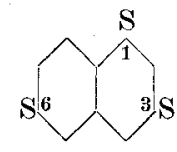

II.

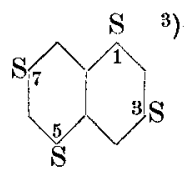

1) Über den Nachweis des 2,1-Amidonaphtols siehe Liehermann, A. 183, 249 (1876); 21 I, 55 (1882) und Diss. H. Brïtsch, Zürich 1921.

2) Proc. of. chem. Soc. 1885-1895.

3) Je nachdem man zu zählen beginnt, kann man diese Derivate auf ganz verschiedene Arten bezeichnen: z. B. $1,3,6=2,5,7=2,4,7$ etc. 
Nun existiert in der Literatur eine Angabe' ${ }^{1}$, welche behauptet, dass neben diesen Säuren noch eine weitere Tetrasulfosäure entstehe, nämlich jene von Senhofer ${ }^{2}$ ), welche erhalten wird, wenn Naphtalin mit konzentrierter Schwefelsäure und Phosphorpentoxyd auf hohe Temperatur $\left(260^{\circ}\right)$ erhitzt werde. Diese Angabe ist leider kritiklos in die Lehrbücher übergegangen, so dass es nicht möglich war, a priori zu entscheiden, ob bei der Einwirkung von rauchender Schwefelsäure zwei oder drei, eventuell mehrere isomere Tetrasulfosäuren entstehen.

Diese Frage ist für den Techniker von Wichtigkeit, da er wissen sollte, was bei der Darstellung z. B. der Amidonaphtol-disulfosäure 1,8,3,6 (H-Säure) für Nebenreaktionen entstehen, und besonders, wie sich die eine, eventuell mehrere Tetrasulfosäuren des Naphtalins gegen Salpetersäure verhalten.

Wir haben daher die verschiedenen in Frage kommenden Sulfosäuren in der Form von Salzen und ihrel: Sulfochloride dargestellt und dabei Folgendes gefunden:

1. Es entsteht bei der energischen Einwirkung von anhydridhaltiger Schwefelsäure auf Naphtalin immer ein Gemisch der 1,3,6-Trisulfosäure und der 1,3,5,7-Tetrasulfosäure.

2. Die 1,3,5,7-Tetrasulfosäure gibt ein Bariumsalz, das dimorph ist. Der Umwandlungspunkt ist ca. $30^{\circ}$. Oberhalb krystallisiert das Salz mit $8 \mathrm{Mol}$. Wasser, unterhalb $30^{\circ}$ mit $14 \mathrm{Mol}$. Wasser (siehe experimenteller Teil). Die beiden Salze geben identische Tetrasulfochloride, Smp. 261-262 ${ }^{\circ} \mathrm{C}$.

3. Neben diesen beiden Sulfoderivaten entstehen keine hochsulfurierten Produkte.

Die Regel von Armstrong und Wynne gilt für das System Naphtalin-Schwefelsäure + Anhydrid ohne jede Einschränkung. Die Angabe des D.R.P. 79054 (By) ist unrichtig. Es kann nur die 1,3,5,7Tetrasulfosäure entstehen.

4. Die Tetrasulfosäure $1,3,5,7$ bildet sich ausserordentlich leicht. Sie ist gegen Salpetersäure vollkommen passiv. Die scheinbaren Verluste bei der H-Säurefabrikation beruhen auf der Bildung der Tetrasulfosäure, neben oxydativer Zerstörung des Naphtalins.

5. Die 1,3,6-Trisulfosäure ist Endprodukt, wie nach der Regel von Armstrong zu erwarten war. Selbst ein grosser Überschuss von $\mathrm{SO}_{3}$ bewirkt keine Abnahme schon gebildeter 1,3,6-Säure. Dagegen

1) D. R. P. 79054 (By).

2) M. 3, $112(1882)$. 
bildet sich bei zu schwachem Oleum, offenbar durch Umlagerung, sehr leicht die 1,3,5,7-Tetrasulfosäure.

6. Um zur 1,3,6-Trisulfosäure zu gelangen, kann man also zwei Wege einschlagen. Entweder über die 2,5-Disulfosäure $(=1,6)$ oder über die 2,7-Disulfosäure $(=3,6)$.
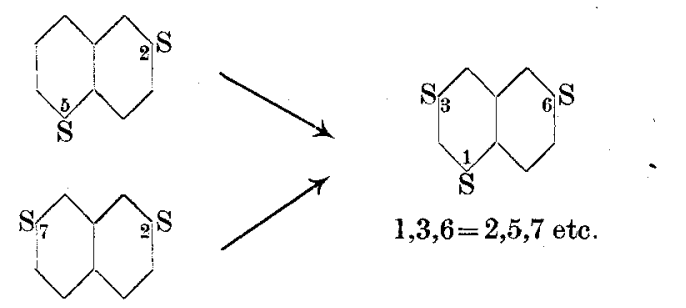

Beide Methoden sind technisch.

7. Als wichtigstes Ergebnis betrachten wir die Tatsache, dass immer neben Tetrasulfosäure wechselnde, oft kleine Mengen Disulfosäure (2,7-Säure; 1,6-Säure) unverändert bleiben, ein Beweis, dass sich diese gerade für die Technik wichtigen Säuren nur schwierig zur 1,3,6-Trisulfosäure weitersulfurieren lassen.

8. Es ist uns nicht gelungen, neben der Nitronaphtalin-trisulfosäure 1,3,6,8 eine andere Nitrosäure zu erkennen, womit wir aber nicht behaupten, dass gar keine isomere Nitrotrisulfosäure gebildet werde. Wir halten aber dafür, dass kein Isomeres, oder dann nur sehr kleine Mengen gebildet werden.

9. Die Nitrotrisulfosäure wurde das erste Mal isoliert und charakterisiert

$$
\left.K=0,095^{1}\right)
$$

\section{Experimenteller Teil.}

Das Naphtalin wurde auf zwei prinzipiell verschiedene Arten sulfuriert. Erstens nach den Angaben von Fierz, Grundlegende Operationen der Farbenchemie (S. 15). Diese Methode (1) wurde dann noch etwas modifiziert (1a), indem ein grosser Oleumüberschuss verwendet wurde.

1. 64 Naphtalin $+70 \mathrm{H}_{2} \mathrm{SO}_{4} 100 \%+30 \mathrm{gr} \mathrm{H}_{3} \mathrm{SO}_{4} 100 \%$ $+230 \mathrm{gr}$ Oleum $64 \% \mathrm{SO}_{3}$;

1a. Wie 1, aber $320 \mathrm{gr}$ Oleum $64 \% \mathrm{SO}_{3}$.

1) Fierz und Weissenbach, Helv. 3, 309 (1920). 
2. $64 \mathrm{gr}$ Naphtalin wurden unter $35^{\circ}$ in $100 \mathrm{H}_{3} \mathrm{SO}_{4} 100 \%+230 \mathrm{gr}$ Oleum $64 \% \mathrm{SO}_{3}$ vorsichtig eingetragen und nach 15 Stunden bei $20^{\circ}$ auf $160^{\circ}$ während einer Stunde erwärmt. Die Mengenverhältnisse waren also im Falle 1 und 2 die gleichen. Genau gleich wurde für $2 \mathrm{a}$ verfahren, wobei die gleichen Mengenverhältnisse wie bei 1 a angewandt wurden.

Aus den Sulfurationsgemischen wurden durch Eingiessen in Wasser und Neutralisation mit Bariumcarbonat die "Gesamt-Bariumsalze" hergestellt. Es wurden erhalten zwischen 263 und 296 gr Bariumsalze, während für das trisulfosaure Naphtalin theoretisch $300 \mathrm{gr}$ pro $1 / 2 \mathrm{Mol}$. Naphtalin zu erwarten ist. Die Ausbeute wäre demnach zwischen 87 und $92 \%$ der Theorie. Der Rest des Naphtalins liegt als Disulfosäure vor, oder ist vollständig zerstört.

\section{Chlorierung der Salze.}

Da sich besonders das Bariumsalz der Tetrasulfosäure ausserordentlich schwierig chlorieren lässt, so wurden immer die Natriumsalze hergestellt. (Umsetzung mit Soda in wässeriger Lösung.)

1 Teil scharf getrocknetes Natriumsalz wurde mit 2 Teilen $\mathrm{PCl}_{5}$ und 3 Teilen $\mathrm{POCl}_{3}$ neun Stunden auf dem kochenden Wasserbade erwärmt. Dann wurde auf Eis gegossen, eine Stunde verrührt, filtriert und bei $40^{\circ}$ getrocknet. Die Ausbeute betrug 85-95\% der Theorie.

\section{Trennung der Chloride.}

Methode 1 und 1 a ergaben Rohchloride vom smp. ca. 170-186 ${ }^{\circ}$. (Viel 1,3,6-Trichlorid Smp.: 190-1910.)

Methode 2 und 2 a ergaben Rohchloride rom Smp. ca. $210-228^{\circ}$. (Viel 1,3,5,7-Tetrachlorid: Smp. 261-2620.)

1. Die trockenen Chloride wurden zuerst mit Eisessig kalt extrahiert, wobei die Dichloride entfernt werlen.

2. Rückstand wurde mit Eisessig (vierfache Menge) aufgekocht und heiss filtriert; es geht 1,3,6-Chlorid in Lösung.

3. Rückstand fast reines 1,3,5,7-Tetrachlorid, als einzige schwerlösliche Komponente. Niemals konnte eine andere Tetrasulfosäure beobachtet werden.

Genaue Angaben über die Trennung finden sich in der Dissertation des Herrn Dr. Fritz Schmid (Zürich 1920). 


\section{$-\quad 385$}

Beispiele von Analysen.

\begin{tabular}{|c|c|c|c|c|c|}
\hline $\begin{array}{l}\text { Methode } 1 \\
\text { (nicht er- } \\
\text { schöpfend) }\end{array}$ & $\begin{array}{l}\text { Rohchlorid } \\
\text { Smp. } \\
175^{\circ}\end{array}$ & $\begin{array}{c}1,3,5,7- \\
\text { Tetrachlorid } \\
5,1 \%\end{array}$ & $\begin{array}{c}1,3,6- \\
\text { Trichlorid } \\
54,8 \%\end{array}$ & $\begin{array}{c}\text { Disulfo- } \\
\text { chloride } \\
\text { Smp. } 148^{0} \\
\text { Smp. } 160^{0} \\
15 \%\end{array}$ & $\begin{array}{c}\text { Isoliertes } \\
\text { Dichlorid } \\
\text { Smp. } \\
147-148^{0} \\
=\text { Gemisch }\end{array}$ \\
\hline Methode la. & $184-186^{0}$ & $19,5 \%$ & $52,1 \%$ & $5,6 \%$ & $\begin{array}{c}158-160^{\circ} \\
\text { Prismen } \\
=2,7-D i- \\
\text { chlorid !! }\end{array}$ \\
\hline $\begin{array}{c}\text { II. } \\
\text { kalt } \\
\text { nicht er- } \\
\text { schöpfend }\end{array}$ & $162-165^{0}$ & $33,8 \%$ & $12 \%$ & $21 \% !$ & $\begin{array}{c}\text { Smp. } \\
147-148\end{array}$ \\
\hline IIa & $215-228^{\circ}$ & $42 \%$ & $12 \%$ & $8 \%$ & $?$ \\
\hline
\end{tabular}

Immer entzieht sich ein Teil der Tetrasäure der vollkommenen Chlorierung. Wir schätzen, dass sich nur $60 \%$ des tetrasulfosauren Natriumsalzes überhaupt vollständig chlorieren lässt, so dass jedenfalls die Zahlen für das Tetrachlorid um 50-60\% zu erhöhen sind (z. B. statt $42 \%$ mindestens $65-70 \%$ ). Versuche mit reinem tetrasulfosaurem Natriumsalz stützen diese Annahme.

Das Bariumsalz der 1,3,5,7-Naphtalintetrasulfosäure wurde wie folgt erhalten: Naphtalin wurde nach einer der angegebenen Methoden sulfuriert (vorzugsweise 2 a). Die sich nach einem Tage ausscheidenden Krystalle wurden über Asbest abgesaugt und mit konzentrierter Schwefelsäure gewaschen, dann der weisse Kuchen, bestehend aus feinen Nadeln, in Wasser gelöst und mit Bariumcarbonat neutralisiert und vom Bariumsulfat abfiltriert. Die klare Lösung ergab beim Eindunsten bei $35^{0}$ als Hauptfraktion derbe, körnige, durchsichtige Krystalle mit sechsseitigen Flächen, die oft zu Zwillingen verwachsen sind. Die Krystalle werden oft $0,5 \mathrm{~cm}$ im Durchmesser gross. Mikroskopisch wurde nur diese Krystallform beobachtet. Die Krystalle sind vollständig luftbeständig.

$2,6438 \mathrm{gr}$ Subst. verloren bei $15 \tilde{5}^{0}$ bis zur Gewichtskonstanz getrocknet

$$
\begin{aligned}
& 0,4211 \text { gr } \mathrm{H}_{2} \mathrm{O}=15,92 \%=7,6 \mathrm{Mol} . \mathrm{H}_{2} \mathrm{O} \\
& \mathrm{Ba}_{2}\left(\mathrm{C}_{10} \mathrm{H}_{4}\left(\mathrm{SO}_{3}\right)_{4}\right)+8 \mathrm{H}_{2} \mathrm{O} \text { Ber. } \mathrm{H}_{2} \mathrm{O} \quad 16,86 \%
\end{aligned}
$$

$0,8268 \mathrm{gr}$ Subst. wasserfrei gaben $0,5354 \mathrm{gr} \mathrm{BaSO}_{4} \mathrm{Ba} 38,11 \%$

Die obige Formel verlangt . . . . . . " $38,22 \%$ 
Wenn die wässerige Lösung des Bariumsalzes bei $20^{\circ}$ verdunstet, so scheiden sich oft 2-4 $\mathrm{cm}$ lange, glasklare, abgestumpfte Prismen aus. Diese verwittern rasch an der Luft. Sie sind identisch mit dem Bariumsalz von Senhofer loc. cit. Frisch analysiert wurden folgende Zahlen erhalten:

$$
\begin{aligned}
& 0,6462 \text { gr Subst. gaben } 0,1547 \text { gr Wasserverlust }=23,94 \% \\
& 0,7420 \text { gr Subst. (wasserfrei) gaben } 0,4764 \mathrm{gr} \mathrm{Ba} \quad \mathrm{Ba} 37,77 \% \\
& \mathrm{Ba}_{2}\left(\mathrm{C}_{10} \mathrm{H}_{4}\left(\mathrm{SO}_{3}\right)_{4}\right)+13 \mathrm{H}_{2} \mathrm{O} \quad \mathrm{H}_{2} \mathrm{O} 23,94 \% \\
& \mathrm{Ba}_{2}\left(\mathrm{C}_{10} \mathrm{H}_{4}\left(\mathrm{SO}_{3}\right)_{4}\right) \quad, \quad 38,22 \%
\end{aligned}
$$

$\mathrm{Da}$, wie aus der Analyse hervorgeht, nicht alles Wasser ausgetrieben werden kann, folgt aus den Zahlen, dass bei $15-20^{\circ}$ das Bariumsalz der 1,3,5,7-Naphtalintetrasulfosäure mit 14 Mol. Wasser krystallisiert.

Der Bimorphismus des vorliegenden salzes kam auch leicht durch gegenseitige Überführung in die beiden Formen bewiesen werden (siehe S. 382).

Die Tetrasulfosäure wird ferner weder mit konzentrierter Salpetersäure noch mit sogen. Mischsäure nitriert, indem aus den Gemischen das unveränderte Bariumsalz wiedergewonnen wurde.

\section{Darstellung der 1,3,6,8-Nitrotrisulfosäure des Naphtalins.}

Wenn man das Nitriergemisch, erhalten z. B. nach ten Angaben von Fierz, „Farbenchemie", S. $15 \mathrm{ff} .$, mit so viel Wasser versetzt, dass eine Säure von ca. $60 \% \mathrm{H}_{2} \mathrm{SO}_{4}$ entsteht, dann scheidet sich beim $\mathrm{Ab}$ kühlen die Säure in der Form von feinen gelben Nadeln aus. Durch wiederholtes Umlösen in ganz wenig Wasser und Sättigen mit Salzsäuregas erhält man die Säure leicht schwefelsänrefrei in der Form von fast weissen, hygroskopischen Närlelchen. T)iese verlieren im Exsiccator einen Teil des Krystallwassers. Frisch hergestellt, enthält die Säure ca. $77 \%$ reine Säure und $23 \%$ Wasser. Im Exsiccator über Calciumchlorid bei $20 \mathrm{~mm}$ Quecksilberlruck, steigt der Gehalt an Nitrotrisulfosäure auf ca. $83,5 \%$.

Die Nitrotrisulfosäure in 4-proz. wässeriger Lösung ist gegen Kochen (fünf Stunden) vollkommen bestiindig, auch unter Zusatz von $5 \mathrm{~cm}^{3}$ konz. Salzsäure auf $50 \mathrm{~cm}^{3}$ Lösung konnte keine Veränderung bemerkt werden. Bariumchlorid zeigt keine freie Schwefelsäure an.

Es wurden vier Salze der Nitrotrisulfosäure dargestellt.

Das Bleisalz bildet orangegelbe, derbe, kurze Nadeln, die warzig verwachsen sind. Das Salz ist mässig löslich in Wasser und verwittert an der Luft. 
Frisch bereitet enthält das Salz 8 Molekeln Krystallwasser.

1. 1,4240 gr Subst. verloren bei $150^{\circ} 0,2364$ gr $\mathrm{H}_{2} \mathrm{O}=16,60 \%=8,0 \mathrm{Mol} \cdot \mathrm{H}_{2} \mathrm{O}$

2. 4,6240 gr lufttrockenes Salz verloren bei $150^{\circ} 0,4506 \mathrm{gr}$ Wasser $=9,74 \%$ oder

3. Im Exsiccator getrocknetes Salz: $=4,3 \mathrm{Mol} . \mathrm{H}_{2} \mathrm{O}$

3,6966 gr Subst. verloren bei $150^{\circ} 0,1952 \mathrm{gr}=5,28 \%=2,22 \mathrm{Mol}$. Wasser

1,8688 gr bei $150^{\circ}$ getrocknetes Bleisalz gaben 1,1632 gr $\mathrm{PbSO}_{4} \quad \mathrm{~Pb} 42,51 \%$ Berechnet für $\left[\mathrm{C}_{10} \mathrm{H}_{4}\left(\mathrm{NO}_{2}\right)\left(\mathrm{SO}_{3}\right)_{3}\right]_{2} \mathrm{~Pb}_{3} \mathrm{~Pb} 43,10 \%$

Das Bariumsalz krystallisiert in gelben, halbkugeligstrahlig angeordneten Nadeln. Das Krystallwasser wird leicht abgegeben.

1. 0,6704 gr Subst. (frisch) verloren bei $150^{\circ} 18,89 \%=8,00 \mathrm{Mol} . \mathrm{H}_{2} \mathrm{O}$

2. $0,8495 \mathrm{gr}$ Subst. (lufttrocken) verloren bei $150^{\circ} 12,21 \%=4,50 \mathrm{Mol} . \mathrm{H}_{2} \mathrm{O}$

32,7938 gr Subst. (exsiccatortrocken) verloren bei $150^{\circ} \quad 9,60 \%=3,61$ Mol. $\mathrm{H}_{2} \mathrm{O}$

1,3606 gr Subst. (trocken) gaben 0,7582 gr $\mathrm{BaSO}_{4} \quad \mathrm{Ba} 33,03 \%$ $\left[\mathrm{C}_{10} \mathrm{H}_{4}\left(\mathrm{NO}_{2}\right)\left(\mathrm{SO}_{3}\right)_{3}\right]_{2} \mathrm{Ba}_{3}$ erfordert , $33,44 \%$

Das Natriumsalz krystallisiert mit 6 Mol. Wasser und ist luftbeständig. Es erscheint in der Form von derben, orangegelben Nadeln oder Prismen.

1,0687 gr Subst. verloren bei $150^{\circ} 18,70 \%$ Wasser $=6,11$ Mol. $\mathrm{H}_{2} \mathrm{O}$

0,5672 gr Subst. (wasserfrei) gaben 0,2535 gr $\mathrm{Na}_{2} \mathrm{SO}_{4} \quad \mathrm{Na} 14,47 \%$

$$
\mathrm{C}_{10} \mathrm{H}_{4}\left(\mathrm{NO}_{2}\right)\left(\mathrm{SO}_{3} \cdot \mathrm{Na}\right)_{3} \text { entspricht } \quad \text {, } 14,40 \%
$$

Das Anilinsalz bildet in heissem Wasser lösliche, feine, weisse Nadeln, die zentrisch angeordnet sind. Es krystallisiert mit 21/2 Mol. Wasser und ist in Alkohol leicht, in Äther schwer löslich.

0,8139 gr Subst. verloren bei $120^{\circ} 6,52 \%=2,66$ Mol. $\mathrm{H}_{2} \mathrm{O}$ 0,8416 gr Subst. verloren bei $120^{\circ} 6,12 \%=2,50 \mathrm{Mol} . \mathrm{H}_{2} \mathrm{O}$

Eine Verbrennung, deren Gewichtszahlen der Kürze halber nicht gegeben werden, gab:

$$
\begin{aligned}
& \mathrm{C}_{10} \mathrm{H}_{4} \mathrm{NO}_{2}\left(\mathrm{SO}_{3} \cdot \mathrm{C}_{6} \mathrm{H}_{5} \mathrm{NH}_{3}\right)_{3}+2 \frac{1}{2} \mathrm{H}_{2} \mathrm{O} \text { Ber. C 45,59 H 4,48 } \quad \mathrm{N} 7,60 \% \\
& \text { Gef. , } 45,71 \text {, 4,34 ,7,71\% }
\end{aligned}
$$

Technisch-chemisches Laboratorium der Eidg. Techn. Hochschule. Zürich, März 1921. 